\title{
Método aproximado para determinar la potencia óptica en una linterna fotónica
}

\section{Approximate method to determine the output optical power in a photonic lantern}

\author{
PALMA-ARGUELLO, Patricia Ixchel $\dagger^{1}$, PÉREZ-SÁNCHEZ, Grethell Georgina*1, MARTÍNEZ- \\ PIÑÓN, Fernando ${ }^{2}$ y HERNÁNDEZ-VALDEZ, Genaro ${ }^{1}$
}

${ }^{1}$ Universidad Autónoma Metropolitana - Unidad Azcapotzalco, Departamento de Electrónica, Av San Pablo Xalpa 180, Reynosa Tamaulipas, Ciudad de México, C.P. 02200

${ }^{2}$ Instituto Politécnico Nacional, Centro de Investigación e innovación Tecnológica, Cerrada Cecati S/N, Col. Sta Catarina, Azcapotzalco, Ciudad de México, C.P. 02250

ID $1^{\mathrm{er}}$ Autor: Patricia Ixchel, Palma-Arguello / ORC ID: 0000-0001-6527-5230, Researcher ID Thomson: G-1187-2019, CVU CONACYT ID: 972079

ID $1^{\text {er }}$ Coautor: Grethell Georgina, Pérez-Sánchez / ORC ID: 0000-0002-5505-6226, Researcher ID Thomson: C-86412018, CVU CONACYT ID: 230934

ID $2^{\mathrm{do}}$ Coautor: Fernando, Martínez-Piñón / ORC ID: 0000-0002-6845-6085, CVU CONACYT ID: 202377

ID $3^{\text {er }}$ Coautor: Genaro, Hernández-Valdez / ORC ID: 0000-0002-1280-3527, CVU CONACYT ID: 35223

DOI: $10.35429 / J O E S .2019 .18 .6 .23 .29$

Recibido 09 Febrero, 2019; Aceptado 28 Marzo, 2019

\section{Resumen}

La astronomía se ha beneficiado de manera significativa con el desarrollo de la tecnología fotónica. Sin embargo, el uso de fibras ópticas monomodo en esta área no es del todo eficiente, esto debido principalmente a que su núcleo, del orden de 8 micras de diámetro, no permite la captación de grandes cantidades de luz. Además, en ciertos estudios astronómicos, se requiere analizar la luz incoherente multimodal proveniente de los astros, particularmente en el rango espectral del infrarrojo cercano. Una solución a estas demandas es el uso de linternas fotónicas, que son dispositivos que funcionan como interfaz entre un conjunto de fibras monomodo y una fibra multimodo, y en cuya transición se obtienen pérdidas ópticas muy bajas. Sin embargo, no existe hasta donde tenemos conocimiento, un método matemático para el análisis del comportamiento de la potencia de salida en las linternas fotónicas. Por lo que, en este trabajo se propone un método numérico de aproximación para determinar la potencia óptica de salida de fibras ópticas monomodo en una linterna fotónica, utilizando la solución de las ecuaciones de eigenvalor, así como la captura espacial de una parte de la distribución de potencia óptica, mediante el extremo de una fibra óptica adelgazada monomodo.

Linterna fotónica, Astronomía, Potencia óptica

\begin{abstract}
Astronomy has benefited significantly from the development of photonic technology. However, the use of single-mode optical fibers in this area is not entirely efficient, this is mainly since its core, of the order of 8 microns in diameter, does not allow the capture of large amounts of light. In addition, in certain astronomical studies, it is required to analyze the multimodal incoherent light coming from the stars, particularly in the spectral range of the near infrared. One solution to these demands is the use of photonic lanterns, which are devices that function as an interface between a set of single-mode fibers and a multimode fiber, and in whose transition very low optical losses are obtained. However, there is not as far as we know, a mathematical method for the analysis of the behavior of the output power in photonic lanterns. Therefore, in this work we propose a numerical approximation method to determine the optical output power of single mode optical fibers in a photonic lantern, using the solution of eigenvalue equations, as well as the spatial capture of a part of the distribution of optical power, through of the end of a monomode tapered optical fiber.
\end{abstract}

Photonic-lantern, Astronomy, Optical power

Citación: PALMA-ARGUELLO, Patricia Ixchel, PÉREZ-SÁNCHEZ, Grethell Georgina, MARTÍNEZ-PIÑÓN, Fernando y HERNÁNDEZ-VALDEZ, Genaro. Método aproximado para determinar la potencia óptica en una linterna fotónica. Revista de Sistemas Experimentales. 2019. 6-18: 23-29

\footnotetext{
* Correspondencia al Autor (Correo electrónico: ggps @azc.uam.mx)

$\dagger$ Investigador contribuyendo como primer Autor
} 


\section{Introducción}

Una linterna fotónica es un dispositivo en el cual una fibra multimodo (MM) se une a un conjunto de fibras monomodo (SM) adelgazadas (“Optical fiber tapers" en inglés. "Taper", en general, se refiere a una estructura geométrica cónica), y cuyo funcionamiento permite el paso de diferentes modos de propagación de la luz al mismo tiempo sin que interfieran entre sí, ya que estos toman un camino separado por las diferentes fibras SM, ver Figura 1(Leon-Saval, Birks, Bland-Hawthorn, \& Englund, 2005; Yerolatsitis \& Leon-saval, 2015).

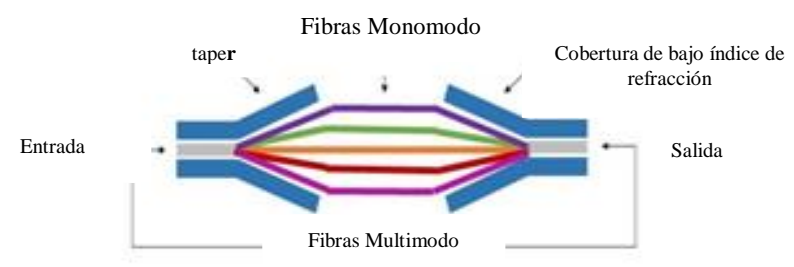

Figura 1 Diagrama esquemático de una linterna fotónica.

En astronomía, las linternas fotónicas se utilizan para la observación en el rango del espectro del infrarrojo cercano y medio, ya que permiten transportar y filtrar información desde el plano focal del telescopio hasta los instrumentos de análisis, con pérdidas muy bajas. Debido a que las fibras MM permiten la transmisión de múltiples modos en el mismo núcleo, la luz incoherente proveniente de los astros puede ser transportada desde el telescopio por la fibra MM hasta la zona de transición con las fibras SM.

Así cada modo de propagación es transmitido en las diferentes fibras SM, y filtrado mediante rejillas de Bragg, para posteriormente ser enviadas por la fibra MM de salida hacia espectrógrafos que analizan la información, como se puede observar en la Figura 2.

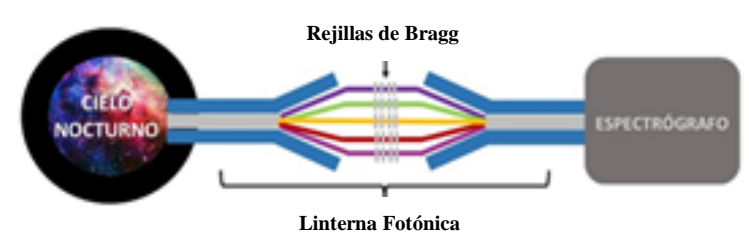

Figura 2 Linterna fotónica como instrumento para la observación en astronomía
Las linternas fotónicas en astronomía permiten realizar mediciones de la luz obtenida en observación, ya que con un solo dispositivo se pueden recolectar y filtrar diferentes bandas de información al mismo tiempo, con pérdidas muy bajas en el proceso, siendo el proceso de filtrado físico por medio de rejillas de Bragg más rápido y directo que el filtrado digital. (Jovanovic, Guyon, Kawahara, \& Kotani, 2017).

De las linternas fotónicas se sabe que, la luz proveniente de la fibra MM excita diferentes modos de propagación en la guía de onda, los cuales se acoplan de manera preferencial a las diferentes fibras monomodo en función de su posición a lo largo de la transición, viajando a través de estas de forma separada. Sin embargo, el proceso exacto mediante el cual cada modo elige una guía de onda, aún no ha sido explorado en su totalidad, ya que los trabajos realizados hasta el momento se enfocan en excitar los modos por separado y analizarlos individualmente (Chala Estrada Roberto Daniel, 2019; González \& Morris, 2019; Miguel Caldas Abril, 2018; Velázquez-benítez et al., 2018).

\section{Objetivo}

El objetivo principal de este trabajo es determinar la distribución de potencia óptica total a partir de la potencia individual de los diferentes modos de propagación excitados en una linterna fotónica vía sus fibras ópticas monomodo de entrada.

\section{Metodología}

En este trabajo se desarrolla un método de simulación para obtener la superposición de modos de propagación de la luz dentro de una fibra óptica MM, la potencia óptica en el área transversal de esta, y la distribución de potencia en una linterna fotónica de pocos modos.

El método desarrollado, se basa en las ecuaciones propuestas por Gloge para los modos aproximados Linealmente Polarizados (LP) (Gloge \& Equation, 1971), con el supuesto que, aunque los modos viajan por la fibra sin interferir entre sí, la intensidad óptica en el área transversal de la misma es la suma de las intensidades de cada modo individual. Además, la distribución de potencia total en el área de transición con las fibras SM es lo que excita la creación de los modos en estas últimas. 
El método de simulación propuesto consiste en los siguientes pasos:

- Obtención de la ecuación de eigenvalor.

- Solución a la ecuación de eigenvalor.

- $\quad$ Superposición de modos y cálculo de la potencia óptica.

Distribución de potencia óptica de salida de la linterna fotónica.

Para llevarlos a cabo, se realiza la simulación de los modos de propagación de la luz que pueden ser excitados y transmitidos en una fibra MM, posteriormente se simula la superposición de dichos modos, basándose en las suposiciones antes mencionadas, y el resultado obtenido se aplica a una potencia de excitación de $1 \mathrm{~mW}$. Finalmente, se simula la distribución de potencia transmitida a un arreglo de siete fibras SM adelgazadas.

Con este método se logra aproximar el comportamiento del campo electromagnético dentro de una fibra MM en múltiples aspectos, como lo son la distribución de campo, la potencia óptica y la intensidad lumínica, lo que es de interés no solo para la astronomía, sino también para el campo de las telecomunicaciones, el diseño de sensores y todas las áreas tecnológicas donde se involucre el uso de fibras ópticas (guías de onda).

\section{Contribución de la investigación}

La contribución principal de este trabajo es el diseño de un método numérico de simulación para determinar la distribución de potencia óptica total a la salida de una linterna fotónica, dependiendo del número de fibras ópticas monomodo a la entrada; lo cual determina también el número de modos excitados. Existen diversos trabajos de investigación sobre el análisis de linternas fotónicas, sin embargo, la determinación teórica de la distribución de la potencia óptica total no ha sido reportada.

\section{Análisis teórico y simulación}

\section{Obtención de la ecuación de Eigenvalor}

Se considera la estructura interna de una fibra multimodo como se muestra en la Figura 3.

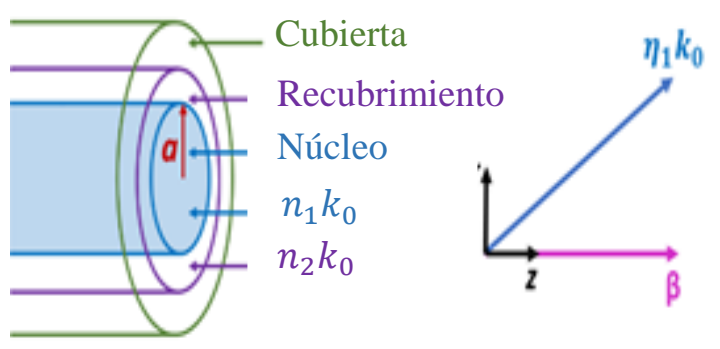

Figura 3 Diagrama de la estructura interna de una fibra óptica multimodo

En donde las ecuaciones paramétricas de la fibra están descritas por:

$$
\begin{aligned}
& u^{2}=a^{2}\left(n_{1}^{2} k_{0}^{2}-\beta^{2}\right) \\
& w^{2}=a^{2}\left(\beta^{2}-n_{2}^{2} k_{0}^{2}\right) \\
& v^{2}=u^{2}+w^{2} \\
& v=\frac{2 \pi}{\lambda} a \sqrt{n_{1}^{2}-n_{2}^{2}}
\end{aligned}
$$

Donde $v$ es la frecuencia normalizada, $u$ y $w$ son los parámetros modales de la guía, $n_{1}$ es el índice de refracción del núcleo, $n_{2}$ es el índice de refracción del revestimiento, a es el radio del núcleo y $\lambda$ es la longitud de onda.

La ecuación de eigenvalor o ecuación característica de la fibra para modos aproximados o modos polarizados linealmente por guiado débil $\left(\mathrm{n}_{1} \approx \mathrm{n}_{2}\right)$ es:

$\frac{u J_{l+1}(u)}{J_{l}(u)}=\frac{w K_{l+1}(w)}{K_{l}(w)}$

Donde $J_{l}$ es la función Bessel de primera clase de orden $l$ y $K_{l}$ es la función Bessel modificada de segunda clase de orden $l$. De las cuales obtenemos las ecuaciones para el campo electromagnético $\mathrm{F}$ en fibras de guiado débil, $\mathrm{r}$ es la coordenada radial el cual varía entre 0 y el radio del recubrimiento. Las funciones están definidas en coordenadas cilíndricas debido a la geometría de la fibra como:

$F_{l}=\frac{J_{l}\left(u \frac{r}{a}\right)}{J_{l}(u)} \cos (l \varphi), 0<r<a$
$F_{l}=\frac{K_{l}\left(w \frac{r}{a}\right)}{K_{l}(w)} \cos (l \varphi), r>a$

Para poder realizar el tratamiento en el lenguaje de programación utilizado, se realiza la conversión a coordenadas rectangulares, por lo que las ecuaciones 6 y 7 quedan como: 
$F_{l}=\frac{J_{l}\left(u \frac{\sqrt{x^{2}+y^{2}}}{a}\right)}{J_{l}(u)} \frac{x}{\sqrt{x^{2}+y^{2}}}$

si $\sqrt{x^{2}+y^{2}} \leq a$.

$F_{l}=\frac{K_{l}\left(w \frac{\sqrt{x^{2}+y^{2}}}{a}\right)}{K_{l}(w)} \frac{x}{\sqrt{x^{2}+y^{2}}}$,

si $\sqrt{x^{2}+y^{2}}>a$

La función $F_{l}$ nos brinda el valor porcentual del campo $\Psi(\mathrm{E}, \mathrm{H})$ en cada par coordenado $(r, \varphi)$, por lo cual 8 y 9 quedan definidas como:

$\Psi=\frac{J_{l}\left(u \frac{\sqrt{x^{2}+y^{2}}}{a}\right)}{J_{l}(u)} \frac{x}{\sqrt{x^{2}+y^{2}}}$,

si $\sqrt{x^{2}+y^{2}} \leq a$

$\Psi=\frac{K_{l}\left(w \frac{\sqrt{x^{2}+y^{2}}}{a}\right)}{K_{l}(w)} \frac{x}{\sqrt{x^{2}+y^{2}}}$,

si $\sqrt{x^{2}+y^{2}}>a$

Para obtener la potencia óptica $P$ se utiliza la siguiente ecuación:

$P \propto \alpha \Psi^{2}$

Donde $\alpha$ es una constante de proporcionalidad.

\section{Solución a la ecuación de eigenvalor}

Los parámetros $u$ y $w$ de la fibra son las soluciones a la ecuación (5), y como estas dependen del radio del núcleo, es de esperarse que, al resolverla, se obtengan varios pares de soluciones para la misma. Es esto lo que da origen a las familias de modos, que en el caso aproximado se denotan como $L P_{l m}$, donde $l$ representa el número correspondiente a la familia, y $m$ el modo individual.

Además, el parámetro $v$ que se obtiene de ellos, nos sirve como indicador del orden y número de modos generados en una fibra dada. Con el fin de resolver la ecuación de eigenvalor para las familias de modos (10 y 11), se '987formuló un código que, utilizando las ecuaciones antes mencionadas, realiza el proceso que se ilustra con el diagrama de flujo de la Figura 4.

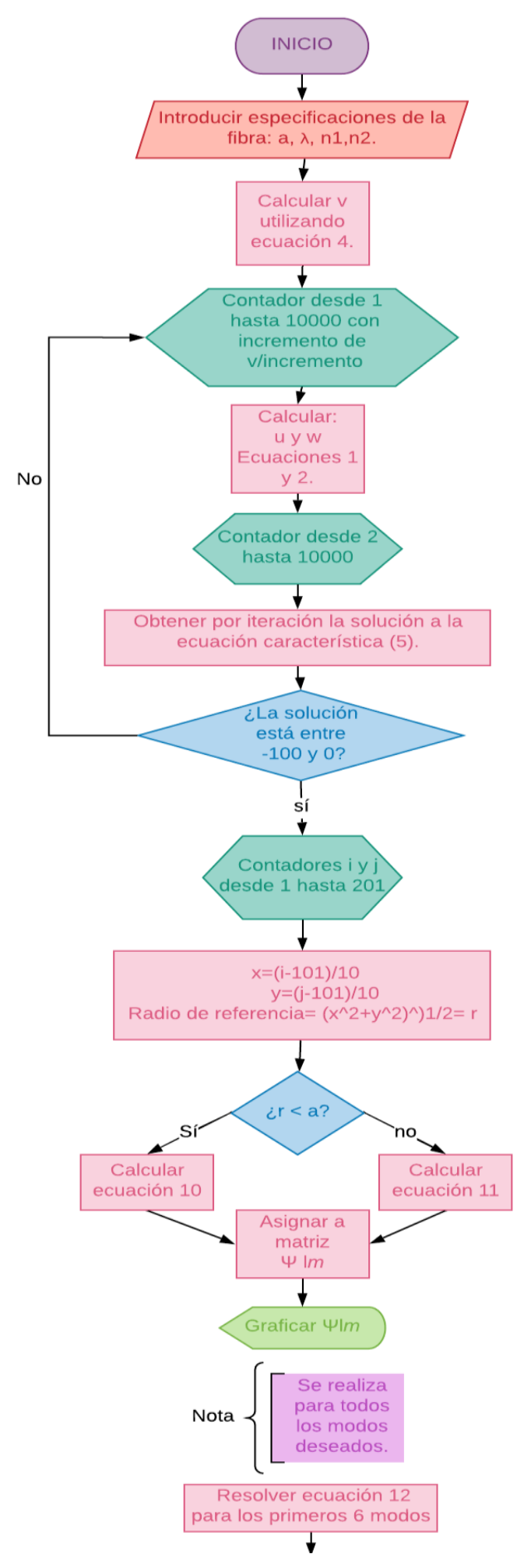




\section{Sumar potencia de los primeros
6 modos \\ Superposición de modos}

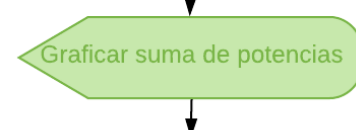

$\downarrow$

Resolver ecuación 12

para los primeros 20 modos

$\downarrow$

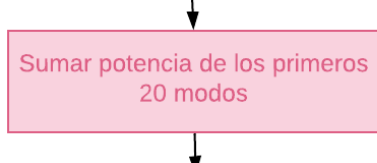

Asignar a matriz Suma de Potencias

$\downarrow$
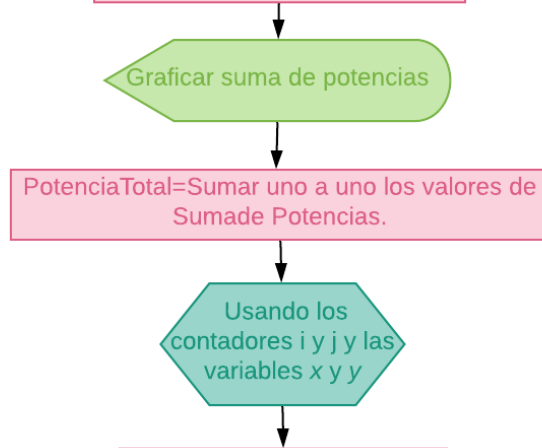

$\checkmark$

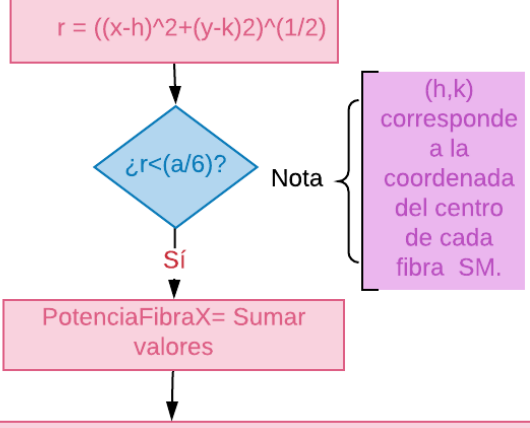

PotenciaFinraXenMicroWatt=PotenciaFibraX/PotenciaTotal

Figura 4 Diagrama de flujo del modelo de solución al problema de la potencia óptica de salida en la fibra MM de una linterna fotónica.

El resultado obtenido en la primera parte del código es una matriz de orden 200x200 formada por los valores de campo $\Psi(\mathrm{E}, \mathrm{H})$ en cada punto. La segunda parte del código se encarga de graficar tridimensionalmente el perfil de campo $\Psi(\mathrm{E}, \mathrm{H})$ y de brindarnos la vista superior de este (ejemplos en Anexo 1), que es de interés para comparar con las reportadas en la enciclopedia de RP Photonics ("RP Photonics Encyclopedia - modes, waveguide, propagation modes, optical fiber, resonator, resonator, field distribution," n.d.).

Para lograr igualar la polarización en las familias de modos impares, se agrega una fase inicial al argumento de $\cos (l \varphi) \rightarrow \cos (l \varphi+\delta)$. También es necesario cambiar el valor de $l$ en las funciones para obtener las matrices de todas las familias de modos.
Una vez calculadas las matrices de campo para cada uno de los modos, estas son elevadas al cuadrado de forma individual con el fin de obtener una matriz de potencia óptica para cada modo, como lo indica la ecuación 12.

Las matrices obtenidas son sumadas con el fin de obtener la matriz de potencia correspondiente a la superposición de los modos. Esta matriz es graficada tridimensionalmente y se puede observar el perfil de la distribución de potencia, así como la vista superior de la misma.

\section{Cálculo de la potencia óptica de salida}

La estructura interna de un conjunto de fibras adelgazadas en el taper de una linterna fotónica, como se puede observar en la Figura 5, las fibras SM se han adelgazado de forma adiabática (con bajas pérdidas) lo suficiente, de tal forma que el núcleo pasa a ser despreciable y la nueva guía de onda es el recubrimiento de la misma, por lo tanto, suponemos sus áreas transversales como las que reciben la potencia óptica de salida para transportar los modos.

Se realiza la suposición de siete fibras SM adelgazadas de igual radio, colocadas en una configuración similar a la de la Figura 5, las cuales son aproximadas a círculos dentro de la matriz de potencia correspondiente a la superposición de los primeros veinte modos de propagación. Geométricamente, se calcula la posición de estos dentro de la matriz, y se suman los valores de la potencia correspondientes a cada uno de los pares ordenados $(x, y)$ que se encuentren dentro del área de dicho círculo.

También se realiza la suma del valor de potencia de todos los puntos contenidos en un círculo, correspondiente al núcleo de la fibra $\mathrm{MM}$, esto con el fin de conocer cuál es el valor total de potencia dentro de la fibra MM y qué proporción de esta es transmitida por cada una de las fibras SM adelgazadas. 


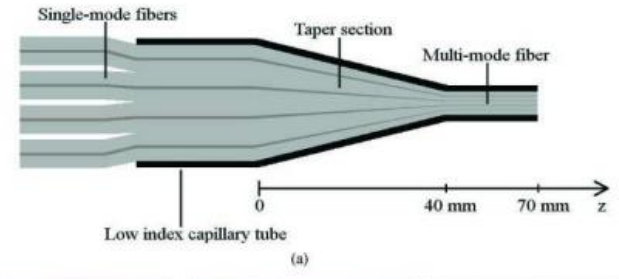

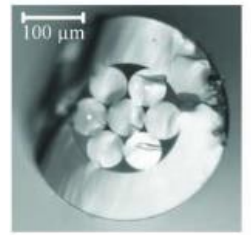

(b)

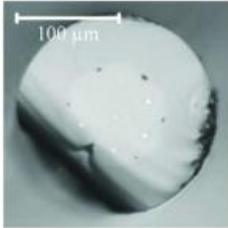

(c)

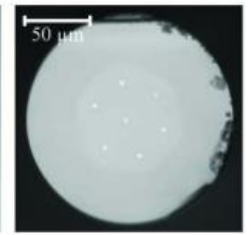

(d)
Figura 5 (a) Ilustración esquemática de una linterna fotónica. (b)-(d) Imagen microscópica de la sección transversal del conjunto de fibras en diferentes posiciones de la transición del taper, a $\mathrm{z}=5 \mathrm{~mm}, \mathrm{z}=20 \mathrm{~mm}$ y $\mathrm{z}=25 \mathrm{~mm}$, respectivamente. Tomada de Efficient multi-mode to single-mode coupling in a photonic lantern.(Noordegraaf, Skovgaard, \& Nielsen, 2009)

Como una primera aproximación, se considera que la potencia total del circulo correspondiente a la fibra MM corresponde a $1000 \mu \mathrm{W}$ de potencia de excitación, y por medio de una simple regla de proporcionalidad, se obtiene la fracción de potencia que se transmite a cada fibra SM. El arreglo de las fibras SM se muestra en la Figura 6.

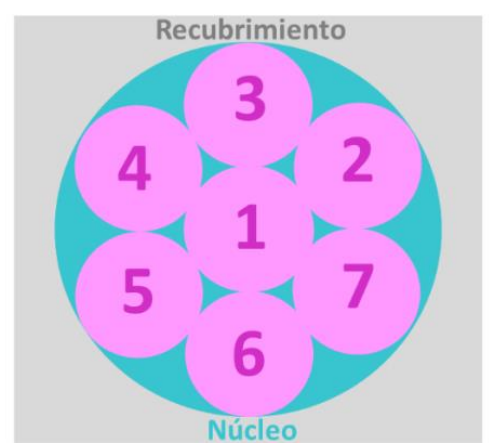

Figura 6 Arreglo espacial de siete fibras SM (círculos morados) conectados al núcleo de la fibra MM que será utilizado en este trabajo

\section{Resultados}

El proceso descrito anteriormente, fue aplicado a la superposición de los primeros seis modos de propagación con la polarización obtenida del código, sin modificar la fase inicial y también con la polarización correspondiente a la reportada por RP Photonics, esto para comparar sus distribuciones de potencia (Gráfico 1). Posteriormente, se realiza el mismo proceso para los primeros veinte modos de propagación (Gráfico 2).

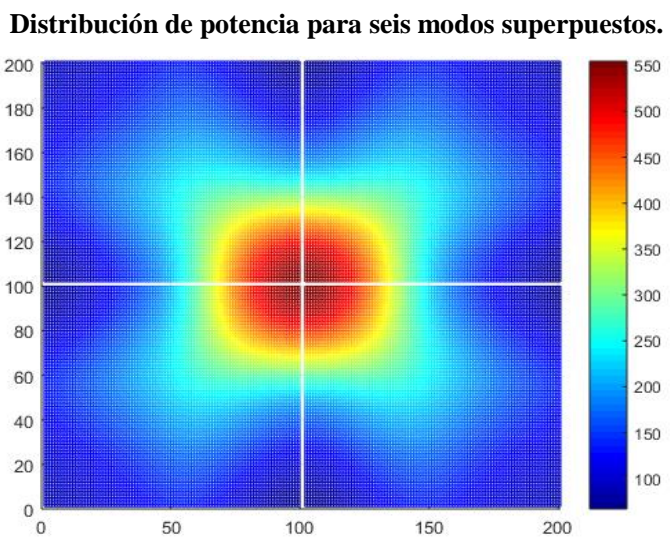

Gráfico 1 Vista superior del perfil de potencia para la superposición de los primeros seis modos de propagación

Distribución de potencia para veinte modos superpuestos.

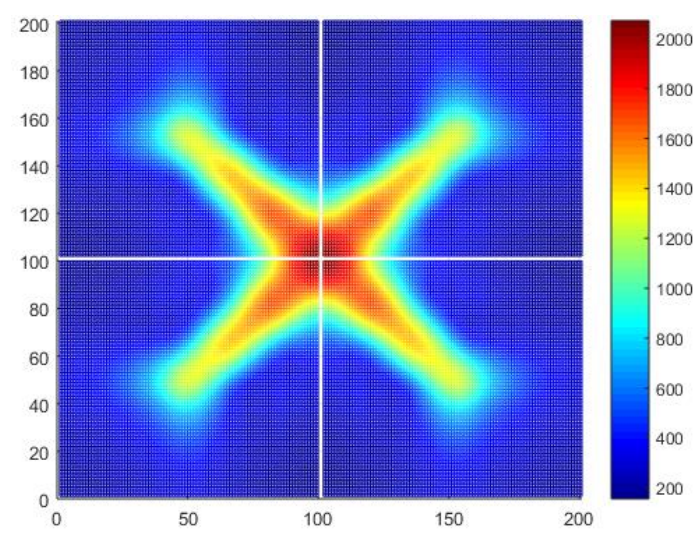

Gráfico 2 Vista superior del perfil de potencia para la superposición de los primeros veinte modos de propagación

Aplicando el proceso descrito en el apartado 2.4 a la superposición de los primeros seis y veinte modos de propagación simulados con nuestro código, se obtienen las potencias ópticas de salida mostradas en la Tabla 1.

\begin{tabular}{|l|r|l|r|}
\hline \multicolumn{2}{|c}{ Primeros 6 modos } & \multicolumn{3}{c|}{ Primeros 20 modos } \\
\hline Fibra & $\begin{array}{l}\text { Potencia } \\
{[\boldsymbol{\mu W}]}\end{array}$ & Fibra & $\begin{array}{l}\text { Potencia } \\
{[\boldsymbol{\mu W}]}\end{array}$ \\
\hline $\mathbf{1}$ & 18.9 & 1 & 26.1 \\
\hline $\mathbf{2}$ & 18.4 & 2 & 22.7 \\
\hline $\mathbf{3}$ & 17.5 & 3 & 18.4 \\
\hline $\mathbf{5}$ & 18.4 & 5 & 22.7 \\
\hline $\mathbf{6}$ & 17.5 & 6 & 18.4 \\
\hline $\mathbf{7}$ & 18.4 & 7 & 22.7 \\
\hline
\end{tabular}

Tabla 1 Potencias ópticas de salida correspondientes a cada una de las siete fibras para seis y veinte modos de propagación

En la tabla 1 se puede observar que, mientras aumenta el número de modos que se propagan por la fibra MM, la potencia óptica de salida total es mayor y su distribución espacial para cada una de las fibras SM es menos uniforme, es decir, la diferencia en la cantidad de potencia que obtiene cada fibra es más grande al aumentar el número de modos.

PALMA-ARGUELLO, Patricia Ixchel, PÉREZ-SÁNCHEZ, Grethel Georgina, MARTÍNEZ-PIÑÓN, Fernando y HERNÁNDEZ-VALDEZ, Genaro. Método aproximado para determinar la potencia óptica en una linterna fotónica. Revista de Sistemas Experimentales. 2019 
Este resultado se puede considerar válido en la generalidad de los casos ya que es producto del aumento en la cantidad de campos superpuestos. También se puede observar, que para las fibras 2, 4, 5 y 7 existe una simetría en la potencia recibida, esto es debido a tres factores: la posición de cada fibra en el arreglo espacial, la cantidad de fibras utilizadas y a la polarización específica de cada modo. Esto puede considerarse un resultado particular, sin embargo, es de esperarse que al variar estos tres factores se observe un cambio en la simetría de distribución, sin embargo, ésta sigue existiendo para cada tipo de configuración que se utilice, aunque de diferente forma.

\section{Conclusiones}

Hemos encontrado un método que permite obtener de forma aproximada y mediante simulación la distribución espacial de potencia óptica generada por la superposición de diferentes modos de propagación de la luz en una fibra MM, y cómo es que ésta se distribuye en un arreglo de $n$ fibras SM. Éste se puede aplicar a cualquier tipo y tamaño de fibra, a diferentes polarizaciones de la luz y al número de modos que se desee analizar; modificando los parámetros de entrada y el tamaño de las matrices. El método obtenido es aplicable no sólo a linternas fotónicas de uso astronómico, sino también para linternas fotónicas aplicadas a las telecomunicaciones.

\section{Trabajo Futuro}

En próximos trabajos se pretende realizar una comparación de los resultados obtenidos con el método desarrollado en este trabajo y los resultados de mediciones experimentales. También se buscará desarrollar un método similar para los modos exactos.

\section{Agradecimientos}

Los autores agradecen a la Universidad Autónoma Metropolitana (UAM) Unidad Azcapotzalco y al Centro de Investigación e Innovación Tecnológica (CIITEC) del Instituto Politécnico Nacional por permitir el uso de sus instalaciones en el desarrollo de este trabajo. Grethell Georgina Pérez Sánchez agradece al CONACyT CB-2016-01 por financiar el proyecto 285600. Fernando Martínez-Piñón agradece al IPN por los recursos facilitados en el proyecto de investigación SIP 20181388.

\section{Referencias}

Chala Estrada Roberto Daniel. (2019). Montaje de un espectrómetro con esfera integradora para la caracterización fotométrica de leds.

Gloge, D., \& Equation, C. (1971). Weakly Guiding Fibers. Applied Optics, 10(10), 2252-2258.

González, D. O., \& Morris, P. A. (2019). Diseño de una antena cilindro parabólica offset en la banda $\mathrm{X}$ para el radar FURUNO, 40, 86-100.

Jovanovic, N., Guyon, O., Kawahara, H., \& Kotani, T. (2017). Application of Multicore Optical Fibers in Astronomy. Optical Fiber Communication Conference, $\quad 8(1), \quad 125-127$. https://doi.org/10.1364/OFC.2017.W3H.3

Leon-Saval, S. G., Birks, T. A., Bland-Hawthorn, J., \& Englund, M. (2005). Multimode fiber devices with single-mode performance. Optics Letters, 30(19), 2545. https://doi.org/10.1364/OL.30.002545

Miguel Caldas Abril. (2018). Desarrollo de una metodología para la estimación de las propiedades térmicas del biodiesel a través de métodos ópticos. Retrieved from http://ring.uaq.mx/bitstream/123456789/1201/1/RI007882. pdf

Noordegraaf, D., Skovgaard, P. M. W., \& Nielsen, M. D. (2009). Efficient multi-mode to single-mode coupling in a photonic lantern. Optics Express, 17(3), 1988-1994.

RP Photonics Encyclopedia - modes, waveguide, propagation modes, optical fiber, resonator, resonator, field distribution. (n.d.). Retrieved June 23, 2019, from https://www.rpphotonics.com/modes.html

Velázquez-benítez, A. M., Antonio-lópez, J. E., Alvarado-zacarías, J. C., Fontaine, N. K., Ryf, R., Chen, H., ... Amezcua-correa, R. (2018). Scaling photonic lanterns for space- division multiplexing. Nature, (January), 1-9. https://doi.org/10.1038/s41598-018-27072-2

Yerolatsitis, S., \& Leon-saval, S. G. (2015). The Photonic Lantern. Advances in Optics and Photonics, (April). https://doi.org/10.1364/AOP.7.000107 